09

\title{
Чувствительность отражательных терагерцовых сенсоров водных растворов
}

\author{
(С) А.Б. Сотский, ${ }^{1}$ М.М. Назаров, ${ }^{2}$ С.С. Михеев, ${ }^{1}$ Л.И. Сотская ${ }^{3}$ \\ ${ }^{1}$ Могилевский государственный университет им. А.А. Кулешова, \\ 212022 Могилев, Беларусь \\ ${ }^{2}$ Национальный исследовательский центр „Курчатовский институт“, \\ 123098 Москва, Россия \\ ${ }^{3}$ Белорусско-Российский университет, \\ 212000 Могилев, Беларусь \\ e-mail: ab_sotsky@mail.ru
}

Поступило в Редакцию 11 июня 2020 г.

В окончательной редакции 22 июля 2020 г.

Принято к публикации 19 августа 2020 г.

Исследована чувствительность сенсоров состава водных растворов, в которых осуществляется регистрация терагерцового сигнала, отраженного от структуры диэлектрическая пленка-раствор. Показано, что максимизация чувствительности достигается при выборе углов падения и частот излучения, обеспечивающем нулевое отражение монохроматического излучения от названной диссипативной структуры. Выяснены условия реализации такого отражения для волн $s$ - и $p$-поляризации. Расчеты подтверждены экспериментально на примере сенсора концентрации глюкозы в воде, в котором использована тонкая кремниевая пластина, отделяющая раствор от воздуха. Установлено, что при использовании метода терагерцовой спектроскопии во временной области предложенная схема сенсора позволяет надежно обнаружить и различить примеси глюкозы концентрации 10 и $30 \mathrm{~g} / 1$.

Ключевые слова: терагерцовый сенсор раствора, закон Брюстера, мода Ценнека, метод терагерцовой спектроскопии во временной области, концентрация глюкозы в водном растворе.

DOI: 10.21883/JTF.2021.02.50368.199-20

\section{Введение}

По мере освоения терагерцового (THz) диапазона частот в биологии и медицине становятся востребованными THz-сенсоры состава жидкостей [1-5]. Это объясняется потребностями спектроскопии биологических жидкостей, поскольку переход от поликристаллического вещества к раствору приводит к существенным изменениям его спектральных характеристик в THz-диапазоне [1,2]. Однако создание таких сенсоров сталкивается с высоким поглощением THz-излучения водой и другими полярными жидкостями. Например, слой воды толщиной $200 \mu \mathrm{m}$ ослабляет интенсивность THz-излучения на два порядка, а слой толщиной $1 \mathrm{~mm}$ практически непрозрачен во всем THz-диапазоне частот. В таких условиях предпочтительно использовать сенсоры отражательного типа, которые более чувствительны к выявлению малых различий в спектральных характеристиках растворов по сравнению с измерением пропускания растворов в кювете [1]. Кроме того, с использованием таких сенсоров принципиально возможно решение важной проблемы неинвазивного контроля концентрации глюкозы в крови [3]. Однако и чувствительность известных сенсоров отражательного типа сравнительно невысока, что объясняется малой протяженностью области взаимодействия зондирующего излучения с исследуемой средой. В настоящей работе показано, что преодо- леть данное ограничение и на этой основе существенно повысить чувствительность THz-спектроскопии водных растворов позволяет использование пленочного сенсора с нулевым отражением. Эффект нулевого отражения имеет аналогию с классическим законом Брюстера, который утверждает равенство нулю коэффициента отражения $r_{p}$ плоской волны $p$-поляризации при ее падении на плоскую границу раздела двух однородных сред с вещественными диэлектрическими проницаемостями $\varepsilon_{a}$ и $\varepsilon_{s}$ со стороны среды с диэлектрической проницаемостью $\varepsilon_{a}$ под углом $b=\operatorname{arctg} \sqrt{\varepsilon_{s} \varepsilon_{a}^{-1}}$, называемым углом Брюстера [6]. В присутствии поглощения в средах и при отклонении границы раздела от идеальной плоской геометрии данный закон строго не выполняется, но если указанные факторы невелики, то отражательная способность $\left|r_{P}\right|^{2}$ имеет минимум при угле падения $\theta_{b} \approx \operatorname{arctg}\left[\sqrt{\operatorname{Re} \varepsilon_{s}\left(\operatorname{Re} \varepsilon_{a}\right)^{-1}}\right]$, который называется псевдобрюстеровским углом [7-9]. Использование в экспериментах углов падения, близких к $\theta_{b}$, приводит к возбуждению быстрых мод Ценнека [10], благодаря чему длина взаимодействия излучения с границей раздела сред, одной из которых является исследуемая среда, значительно превосходит длину волны излучения. В результате значения $\theta_{b},\left|r_{p}\left(\theta_{b}\right)\right|$ и отношение $r_{p}\left(\theta_{b}\right) r_{s}\left(\theta_{b}\right)$ $\left(r_{s}\right.$ - коэффициент отражения плоской волны $s$-поляризации) оказываются весьма чувствительными к параметрам границы раздела [7-9]. Это объясняет широкое 
использование псевдобрюстеровских углов при решении обратных задач эллипсометрии и спектрофотометрии поверхности [7,9]. Но при наличии значительного поглощения электромагнитного излучения исследуемым раствором псевдобрюстеровский угол утрачивает практическое значение (см. ниже). Тем не менее, как установлено в [11], в диссипативных структурах пленкаподложка при определенных углах падения излучения на структуру и на определенных частотах может быть реализовано нулевое отражение от структур волн как $s$-, так и $p$-поляризации, причем в последнем случае возможен предельный переход к закону Брюстера. Режим нулевого отражения эквивалентен возбуждению быстрых длиннопробежных мод структуры, являющихся аналогами мод Ценнека. Поэтому при использовании THz-сенсоров отражательного типа с конфигурацией пленка-исследуемый раствор можно надеяться на существенное увеличение чувствительности к спектральным характеристикам раствора. Ниже этот факт подтвержден теоретически и экспериментально. В частности, методом THz-спектроскопии во временной области (метод THz-TDS [12]) осуществлено надежное детектирование концентрации глюкозы в воде на уровне $10 \mathrm{~g} / 1$, что с использованием других известных THz-сенсоров водных растворов проблематично.

\section{1. Чувствительность отражательных THz-сенсоров водных растворов}

Рассмотрим сенсоры отражательного типа, в которых методом THz-TDS измеряется дисперсия отношения $r_{\alpha} / r_{\alpha}^{(0)}$. Здесь $r_{\alpha}$ и $r_{\alpha}^{(0)}=\left.r_{\alpha}\right|_{C=0}-$ комплексные коэффициенты отражения плоских волн $p$ - $(\alpha=p)$, либо $s$-поляризации $(\alpha=s)$ от пленки толщины $d$, контактирующей с водой, содержащей примесный компонент концентрации $C$, и с чистой водой $(C=0)$. Излучение падает на пленку под углом $\theta$ со стороны среды с вещественной диэлектрической проницаемостью $\varepsilon_{a}$. Пленка и раствор разделены плоской границей и имеют комплексные диэлектрические проницаемости $\varepsilon_{f}$ и $\varepsilon_{s}$ соответственно.

Согласно [11]:

$$
\begin{gathered}
r_{\alpha}=F_{\alpha} \Phi_{\alpha}^{-1} \\
F_{p}, \Phi_{p}=\left[1 \mp h_{s} \varepsilon_{a}\left(h_{a} \varepsilon_{s}\right)^{-1}\right] \cos \left(k_{0} \sigma d\right) \\
+i\left[h_{s} \varepsilon_{f}\left(\sigma \varepsilon_{s}\right)^{-1} \mp \sigma \varepsilon_{a}\left(h_{a} \varepsilon_{f}\right)^{-1}\right] \sin \left(k_{0} \sigma d\right) \\
F_{s}, \Phi_{s}=\left(1 \mp h_{s} h_{a}\right)^{-1} \cos \left(k_{0} \sigma d\right) \\
+i\left(h_{s} \sigma^{-1} \mp \sigma h_{a}^{-1}\right) \sin \left(k_{0} \sigma d\right)
\end{gathered}
$$

где $h_{a, s}=\sqrt{\varepsilon_{a, s}-\beta^{2}}, \quad \sigma=\sqrt{\varepsilon_{f}-\beta^{2}}\left(\operatorname{Re}\left(h_{a, s}, \sigma\right) \geq 0\right)$, $k_{0}=2 \pi f c^{-1}-$ волновое число вакуума, $c-$ скорость света, $\beta=\sqrt{\varepsilon_{a}} \sin \theta-$ проекция безразмерного волнового вектора падающей волны на плоскость пленки.
В качестве параметров, характеризующих чувствительность сенсора, примем производные

$$
\left(\partial \rho_{\alpha} / \partial A\right)_{A=0}, \quad\left(\partial \varphi_{\alpha} / \partial A\right)_{A=0},
$$

где $\left.\rho_{\alpha}=\mid r_{\alpha} / r_{\alpha}^{(0)}\right), \varphi_{\alpha}=\arg \left(r_{\alpha}\right)-\arg \left(r_{\alpha}^{(0)}\right), A-$ некий коэффициент, пропорциональный $C$. Для диэлектрической проницаемости раствора воспользуемся моделью

$$
\begin{aligned}
\varepsilon_{s}=2.5 & +\frac{75(1-A)}{1+i 19 \pi f}+\frac{1.47}{1+i 0.46 \pi f} \\
& +\frac{32}{28.09-f^{2}+i 5.35 f},
\end{aligned}
$$

где частота $f$ берется в $\mathrm{THz}, A=K C$, множитель $K$ определяется типом примесного компонента [13].

На рис. 1 сопоставлены графики угловой зависимости чувствительности отражательных сенсоров трех типов, рассчитанные на основании (1)-(3), (5).

Здесь использованы обозначения

$$
\begin{gathered}
M_{\rho}^{(\alpha)}=\log _{10}\left(\max _{f}\left|\frac{\partial \rho_{\alpha}}{\partial A}\right|_{A=0}\right), \\
M_{\varphi}^{(\alpha)}=\log _{10}\left(\max _{f}\left|\frac{\partial \varphi_{\alpha}}{\partial A}\right|\right),
\end{gathered}
$$

где максимумы величин определяются на интервале $0.1 \leq f \leq 1 \mathrm{THz}$.

Рис. 1, $a$ соответствует простейшему сенсору, в котором THz-сигнал отражается от плоской границы раздела воздух-раствор $\left(\varepsilon_{a}=1, d=0 \mu \mathrm{m}\right)$, рис. $1, b-$ сенсору, использующему эффект нарушенного полного внутреннего отражения (НПВО) THz-сигнала от границы раздела основание кремниевой призмы - раствор $\left(\varepsilon_{a}=3.42^{2}, d=0 \mu \mathrm{m}\right)[13]$, рис. $1, c, d-$ предлагаемым сенсорам, в которых $\mathrm{THz}-$ сигнал, падающий из воздуха, отражается от структуры диэлектрическая пленка толщины $d$-раствор. Рис. $1, c$ относится к пленке из высокоомного кремния (пленка $\left.\mathrm{Si}_{1}\right)$ с $\varepsilon_{f}=n_{f}^{2}$, где $n_{f}=3.42-i 0.005$ [14], рис. $1, d-$ к пленке из полипропилена (пленка РP) с $\varepsilon_{f}=2.247-i 0.0036$ [15]. Толщина обеих пленок $d=240 \mu \mathrm{m}$.

Согласно рис. $1, a, b$, чувствительность известных отражательных сенсоров растворов незначительна. Из сравнения рис. $1, a$ и $b$ следует также несколько более высокая чувствительность сенсора, использующего эффект НПВО и преимущество использования волн $p$ поляризации.

Кардинально решить проблему максимизации чувствительности THz-сенсоров водных растворов позволяет использование пленки, отделяющей раствор от воздуха (рис. $1, c, d)$. Здесь существенную роль играют поляризация излучения и диэлектрическая проницаемость пленки. Для высокопреломляющей пленки $\mathrm{Si}_{1}$ в случае использования волн $p$-поляризации можно выделить две области с экстремально высокой чувствительностью сенсора по обоим критериям (4) в диапазонах 

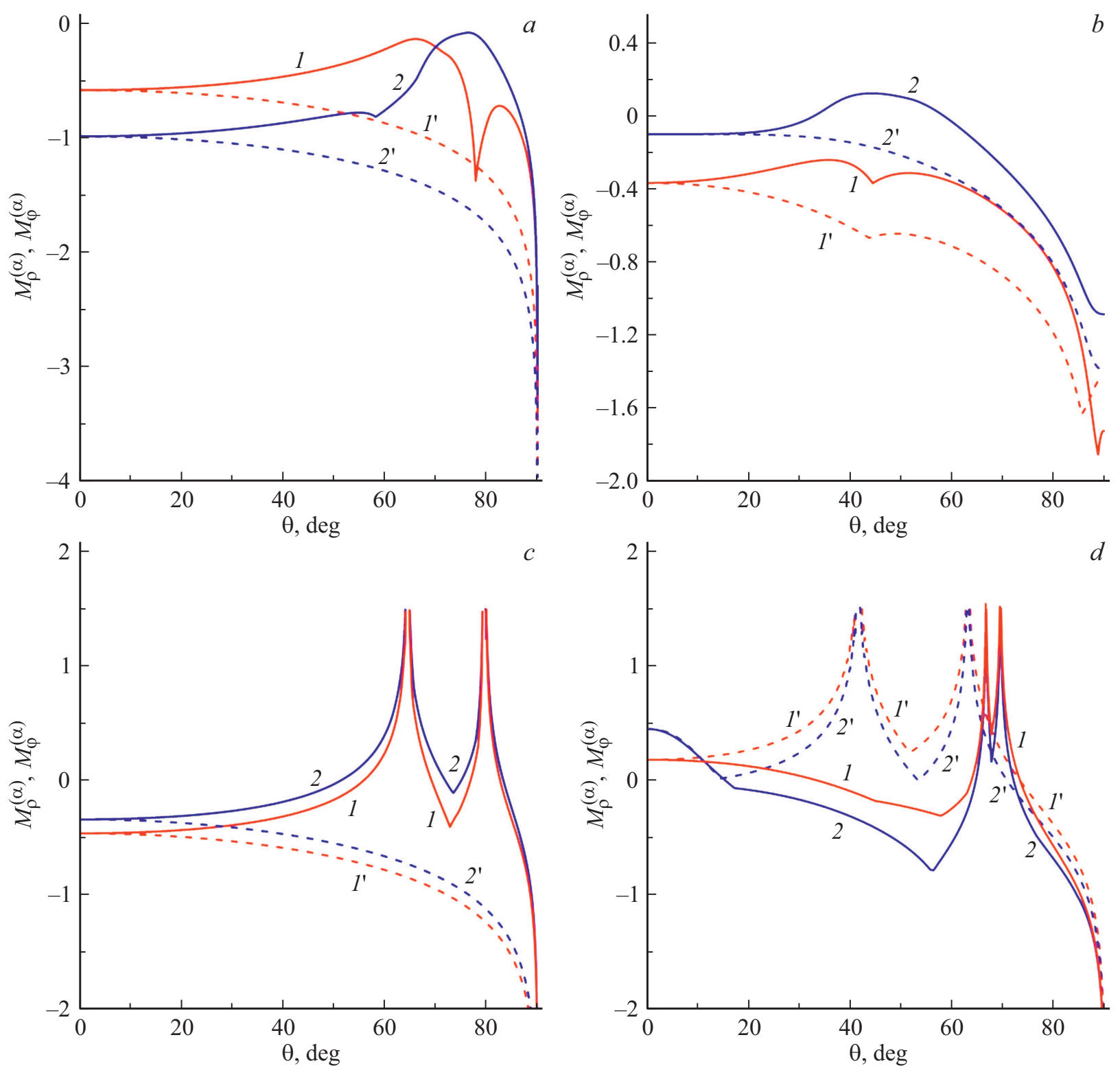

Рис. 1. Угловые зависимости чувствительностей известных $(a, b)$ и предлагаемых $(c, d)$ THz-сенсоров водных растворов отражательного типа. Сплошные кривые: $1-M_{\rho}^{(p)}(\theta), 2-M_{\rho}^{(p)}(\varphi)$, штриховые кривые $1^{\prime}-M_{\rho}^{(s)}(\theta), 2^{\prime}-M_{\rho}^{(s)}(\varphi)$.

углов падения $64.14^{\circ} \leq \theta \leq 64.84^{\circ}$ и $79.21^{\circ} \leq 79.99^{\circ}$ (рис. 1,c). При использовании низкопреломляющей пленки РР аналогичный результат имеет место для волн как $p$-, так и $s$-поляризации (рис. $1, d$ ). Здесь для $s$-волн максимумы чувствительности наблюдаются в диапазонах $40.83^{\circ} \leq \theta \leq 42.24^{\circ}$ и $62.6 \leq \theta \leq 63.54^{\circ}$, а для $p$-волн - в диапазонах $66.61^{\circ} \leq \theta \leq 66.73^{\circ}$ и $69.4 \leq \theta \leq 69.55^{\circ}$. Формально данные особенности следуют из выражения

$$
\left(\frac{d \rho_{\alpha}}{d A}+i \frac{d \varphi_{\alpha}}{d A}\right)_{A=0}=\frac{1}{r_{\alpha}^{(0)}}\left(\frac{\partial r_{\alpha}}{\partial A}\right)_{A=0}
$$

и объясняются наличием в указанных областях нулей функций $r_{\alpha}^{(0)}(\theta, f)$.
Для исследования названных нулей заметим, что комплексное уравнение $r_{\alpha}(\theta, f)=0$, или эквивалентное ему $r_{\alpha}(\beta, f)=0$ при любом $A$ сводится к системе двух вещественных уравнений

$$
\begin{gathered}
L=R_{k}, \\
k_{0} d \operatorname{Re} \sigma=R_{k},
\end{gathered}
$$

где $R_{k}=\operatorname{Re}[\operatorname{Arctg}(z)], L=\operatorname{Re} \sigma(\operatorname{Im} \sigma)^{-1} \operatorname{Im}[\operatorname{Arctg}(z)]$,

$$
\begin{gathered}
z=i\left(h_{s} \varepsilon_{s}^{1}-h_{a} \varepsilon_{a}^{-1}\right) \sigma \varepsilon_{f}^{-1}\left[\left(\sigma \varepsilon_{f}^{-1}\right)^{2}\right. \\
\left.-h_{a} h_{s}\left(\varepsilon_{a} \varepsilon_{s}\right)^{-1}\right]^{-1}(p \text {-волны }), \\
z=i\left(h_{s}-h_{a}\right) \sigma\left[\sigma^{2}-h_{a} h_{s}\right]^{-1}(s \text {-волны }),
\end{gathered}
$$




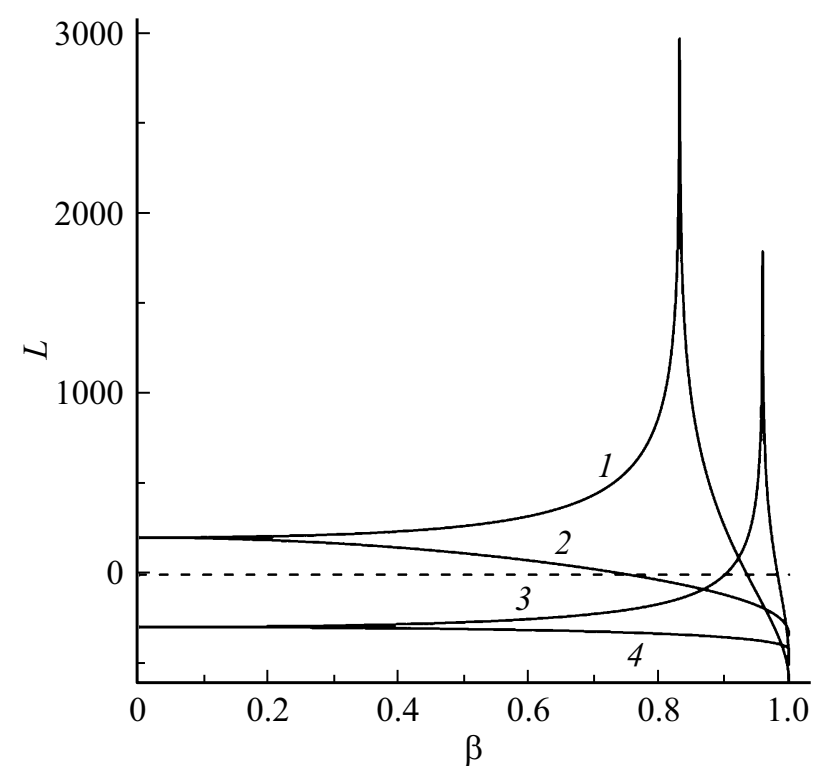

Рис. 2. Зависимости $L(\beta)$ на частоте $f=0.5 \mathrm{THz}$ для РР- $(1,2)$ и $\mathrm{Si}_{1}$-пленок $(3,4)$, контактирующих с водой. Кривые $1,3-p$ волны, $2,4-s$-волны. Штриховая линия - нулевой уровень $L$.

и выбраны ветви бесконечнолистной функции $\operatorname{Arctg}(z)$

$$
\begin{aligned}
\operatorname{Re}[\operatorname{Arctg}(z)]= & 0.5\left\{\operatorname{Arctg}\left[2 x\left(1-|z|^{2}\right)^{-1}\right]\right. \\
& \left.+\pi \operatorname{sign}(x) H\left(|z|^{2}-1\right)\right\}+k \pi,
\end{aligned}
$$

$\operatorname{Im}[\operatorname{Arctg}(z)]=0.25 \operatorname{In}\left\{\left[(1+y)^{2}+x^{2}\right]\left[(1-y)^{2}+x^{2}\right]^{-1}\right\}$,

где $x=\operatorname{Re}(z), y=\operatorname{Im}(z), H(a)=1$ при $a \geq 0, H(a)=0$ при $a<0, k=0,1, \ldots$

Предположим вначале, что частота $f$ фиксирована. Тогда неизвестными в (6), (7) являются $\beta$ и $d$. Ключевую роль играет решение относительно $\beta$ уравнения (6), свойства которого определяются значениями $\varepsilon_{f}$ и $\varepsilon_{s}$. Последующее обращение в тождество уравнения (7) сводится к прямому расчету $d$.

Особенности функции $L(\beta)$ на фиксированной частоте иллюстрирует рис. 2.

В случае $p$-волн график $L(\beta)$ имеет острый максимум при аргументе

$$
\beta=\beta_{m}=\sqrt{\varepsilon_{a} \operatorname{Re}\left(\varepsilon_{f}\right)\left[\operatorname{Re}\left(\varepsilon_{a}+\varepsilon_{f}\right)\right]^{-1}},
$$

который соответствует псевдобрюстеровскому углу падения для границы раздела воздух-материал пленки. Значение $L\left(\beta_{m}\right)$ монотонно возрастает по мере уменьшения $\left|\operatorname{Im} \varepsilon_{f}\right|$, причем $L\left(\beta_{m}\right) \rightarrow \infty$, если $\operatorname{Im} \varepsilon_{f} \rightarrow 0$. Для кремния и полипропилена $L\left(\beta_{m}\right)=1807$ и $L\left(\beta_{m}\right)=2967$ соответственно. В диапазоне $0 \leq \beta \leq \beta_{m}$ функция $L(\beta)$ является монотонно возрастающей, $а$ в диапазоне $\beta_{M}<\beta \leq 1-$ монотонно убывающей. При этом
$L(1)<0$, а знак $L(0)$ зависит от диэлектрических проницаемостей сред. В частности, для слабо поглощающих сред

$$
\operatorname{sign}[L(0)]=\operatorname{sign}\left[\sqrt{\operatorname{Re}\left(\varepsilon_{a}\right) \operatorname{Re}\left(\varepsilon_{s}\right)}-\operatorname{Re}\left(\varepsilon_{f}\right)\right] .
$$

Из (8) следует, что функция $R_{k}(\beta)$ удовлетворяет неравенствам

$$
(k-0.5) \pi \leq R_{k}(\beta) \leq(k+0.5) \pi
$$

и имеет единственный разрыв первого рода при $\beta \approx \beta_{m}$. В результате при рассмотрении $p$-волн система уравнений (6),(7) имеет два конечных дискретных набора решений для $\beta$ и $d$. Эти решения мы обозначим через $\beta_{p k}^{(l)}$ и $d_{p k}^{(l)}$, где $l=1,2-$ номер набора. Набор с $l=1$ относится к области $0 \leq \beta \leq \beta_{m}$, а набор с $l=2-$ к области $\beta_{m}<\beta \leq 1$. Решения из обоих наборов различаются числами $k$ в (8), расположенными в диапазонах $k_{p \min }^{(l)} \leq k \leq k_{p \max }^{(l)}$. Значения $d_{p k}^{(l)}$ удовлетворяют неравенствам $d_{p \min }^{(l)} \leq d_{p k}^{(l)} \leq d_{p \max }^{(l)}$, где $d_{p \min }^{(l)}=d_{p k}^{(l)}$ при $k=k_{p \min }^{(l)}$, $d_{\max }^{(l)}=d_{p k}^{(l)}$ при $k=k_{p \max }^{(l)}$. Согласно (9) и рис. 2, для РР пленки $L(0)>0, k_{p \min }^{(1)}=65, d_{p \min }^{(1)}=1.31 \mathrm{~cm}, k_{p \min }^{(2)}=1$, $d_{p \min }^{(2)}=226.7 \mu \mathrm{m}, k_{p \max }^{(1),(2)}=945, d_{p \max }^{(1),(2)}=22.7$. Для пленки $\mathrm{Si}_{1} L(0)<0, k_{p \min }^{(1)}=0, d_{p \min }^{(1)}=9.46 \mu \mathrm{m}, k_{p \min }^{2}=1, d_{p \min }^{(2)}=$ $=55.05 \mu \mathrm{m}, k_{p \max }^{(1)}=570, d_{p \max }^{(1)}=5.21 \mathrm{~cm}, k_{p \max }^{2}=569$, $d_{p \max }^{(2)}=5.2 \mathrm{~cm}$.

Заметим, что полученные предельные значения толщин пленок $d_{p \max }^{(1),(2)}=22.7 \mathrm{~cm}$ и $d_{p \max }^{(2)}=5.2 \mathrm{~cm}$ весьма велики. Их следует расценивать как формальный результат строгого решения уравнений (6), (7). Практический же интерес представляют толщины пленок, сопоставимые с длиной волны THz-излучения, находящиеся в найденных выше диапазонах (см. далее).

Для $s$-волн функция $L(\beta)$ является монотонно убывающей. Ее максимальное значение $L(0)$ совпадает с $L(0)$ для $p$-волн, а $L(1)<0$. Очевидно, что при $L(0)<0$ система (6), (7) не имеет решений с $d>0$. Таким образом, в случае кремниевой пленки уравнение $r_{\alpha}(\beta, f)=0$ может быть удовлетворено только для $p$-волн. В то же время для РР пленки $L(0)>0$. Как ясно из рис. 2 и неравенств (10), в этом случае для $s$-волн существует единственный набор решений системы (6), (7) для $\beta$ и $d$, отвечающих различным $k$. Эти решения мы обозначим через $\beta_{s k}$ и $d_{s k}$. Их набор дополняет набор с $l=1$ для $p$-волн со стороны меньших значений $d$. Минимальное значение $d_{s k}$, равное $d_{s 0}=97.25 \mu \mathrm{m}$, имеет место при $\beta_{s 0}=0.751$, а максимальное значение $d_{s k}$ совпадает $\mathrm{c} d_{p \min }^{(1)}$.

Рис. 3, где $b_{p k}^{(l)}=\arcsin \left(\beta_{p k}^{(l)}\right), \quad b_{s k}=\arcsin \left(\beta_{s k}\right)$, иллюстрирует частотную зависимость решений уравнений (6), (7), принадлежащих указанным выше наборам.

Представляет интерес определение углов падения $\theta$ и частот $f$, соответствующих нулевому отражению излу- 

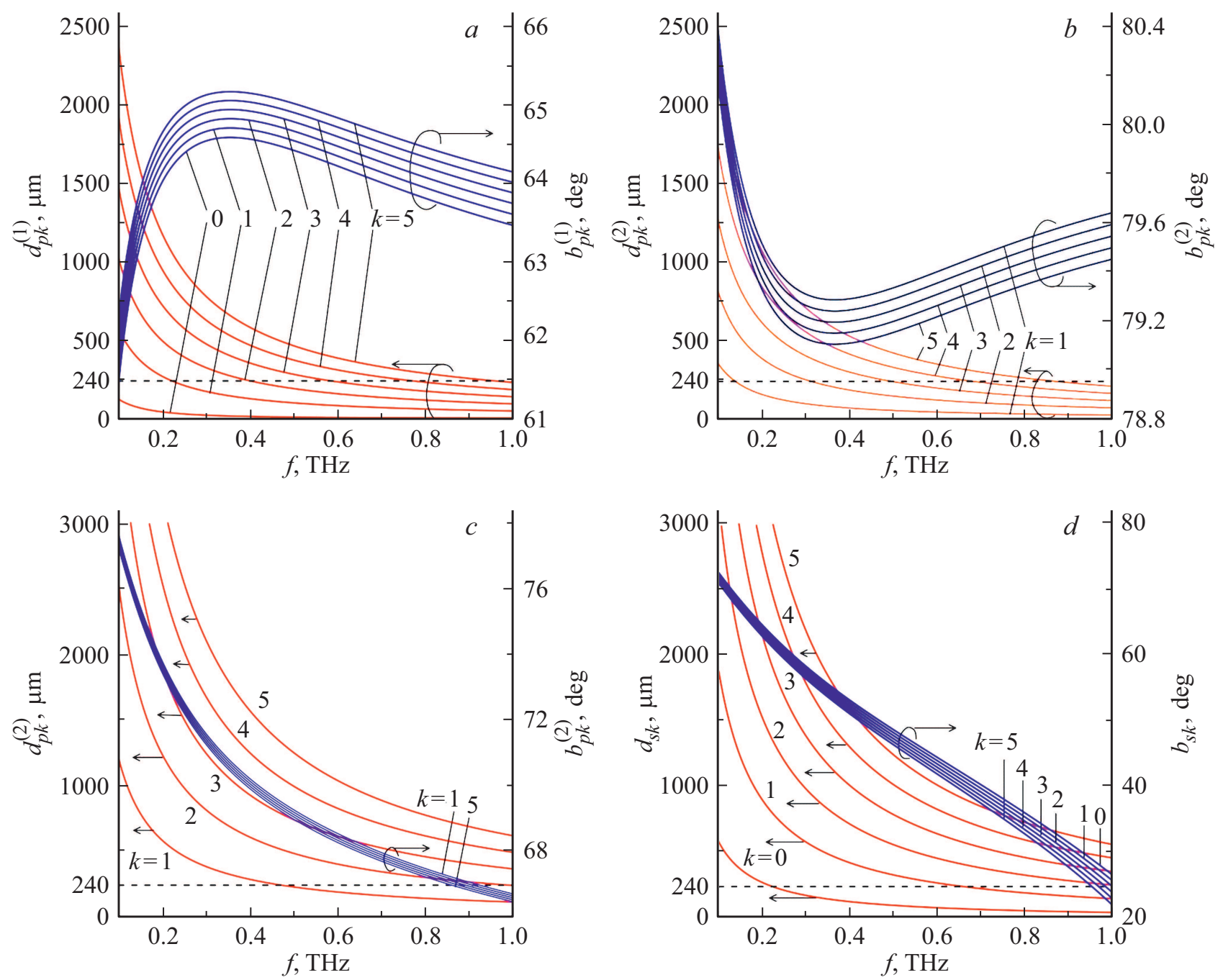

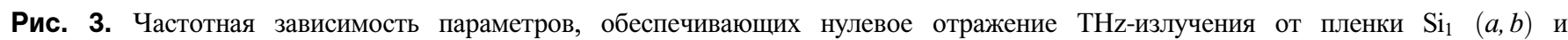
пленки РP $(c, d)$, контактирующих с водой $(A=0)$.

чения от сенсора, при условии задания толщины пленки $d$. В этом случае система уравнений $(6),(7)$ должна решаться относительно $\beta$ и $f$. Как видно из рис. 3, зависимости $d_{p k}^{(l)}(f)$ и $d_{s k}(f)$, отвечающие различным $k$, являются монотонными. Это означает, что уравнение (7) после подстановки в него функции $\beta(f)$, определенной из $(6)$, имеет единственное решение для $f$. Обозначим такие решения для волн $p$-поляризации через $f_{p k}^{(l)}$, а для волн $s$-поляризации - через $f_{s k}$, где индексы имеют такой же смысл, как и в определенных выше величинах $\beta_{p k}^{(l)}=\beta\left(f_{p k}^{(l)}\right), \beta_{s k}=\beta\left(f_{s k}\right)$.

Согласно рис. 3 , пары $f_{p k}^{(l)}, b_{p k}^{(l)}$, для $p$-волн и $f_{s k}, b_{s k}$ для $s$-волн составляют дискретные множества. Например, из рис. $3, a, b$ следует, что при $d=240 \mu \mathrm{m}$ на промежутке $0.1 \leq f \leq 1 \mathrm{THz}$ в каждом из наборов решений для волн $p$-поляризации имеется 5 пар значений $f_{p k}^{(1)}, b_{p k}^{(1)}$, причем $b_{p k}^{(1)}$ находятся в угловых интервалах максимальной чувствительности (рис. $1, c)$. Аналогичная корреляция имеет место между рис. $3, c, d$ и рис. $1, d$.
Округленные значения $f_{p k}^{(1)}, b_{p k}^{(1)}$ для сенсора с пленкой $\mathrm{Si}_{1}$ (рис. 1,a) и $f_{s k}, b_{s k}$ для сенсора с пленкой PP (рис. $3, d$ ), использованные в дальнейших расчетах, приведены в таблице.

На рис. 4 представлены зависимости $\rho_{\alpha}(f)$, исследование которых методом THz-TDS предпочтительнее по сравнению с зависимостями $\varphi_{\alpha}(f)$ ввиду высокой чувствительности последних к погрешностям юстировки сенсора и неоднозначного определения фазовых характеристик THz-сигналов. Расчеты выполнены на основании (1)-(3), (5) при значении $A=0.0074$, которое относится к водному раствору глюкозы концентрации $10 \mathrm{~g} / 1$ [13]. Углы падения излучения на сенсоры выбраны равными табличным $b_{p 1}^{(1)}$ и $b_{s 0}$.

На рис. 4 виден резкий рост чувствительности сенсоров к примеси в растворе при стремлении $f$ к $f_{p 1}^{(1)}$, либо к $f_{s 0}$. Физическая причина данного эффекта состоит в том, что $b_{p k}^{(1)}$, либо $b_{s k}$ являются углами фазового синхронизма падающей на пленку сенсора плоской 

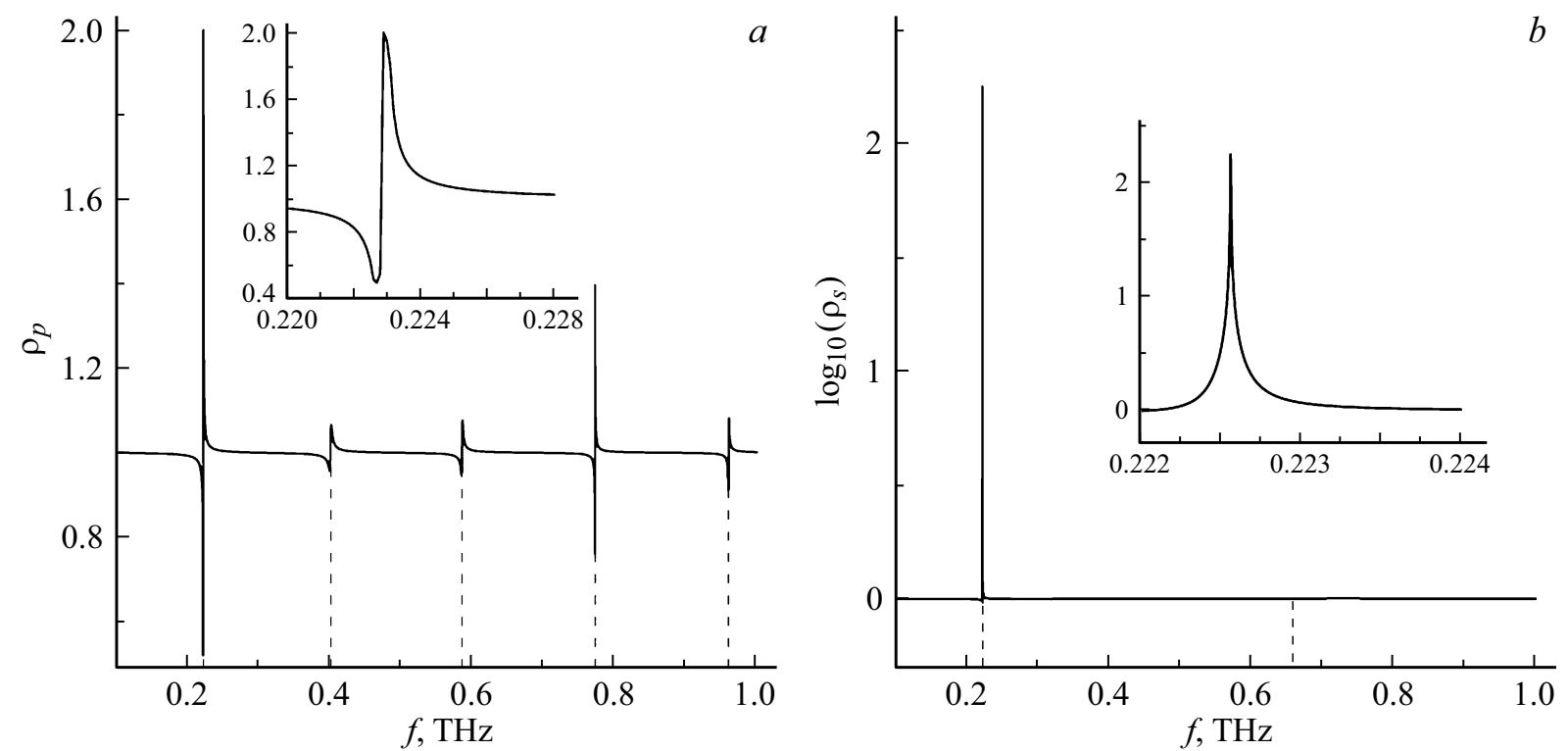

Рис. 4. Зависимости $\rho_{\alpha}(f)$ для сенсоров с пленкой $\mathrm{Si}_{1}$ толщины $d=240 \mu \mathrm{m}$ при $\theta=b_{p 1}^{(1)}=64.32^{\circ}(a)$ и с пленкой РР толщины $d=240 \mu \mathrm{m}$ при $\theta=b_{s 0}=63.09^{\circ}(b)$ в нормальном (диапазон $0.1 \leq f \leq 1 \mathrm{THz}$ ) и уменьшенных (вставки - диапазоны $0.22 \leq f \leq 0.228 \mathrm{THz}$ и $0.222 \leq f \leq 0.224 \mathrm{THz})$ частотных масштабах. Штриховые линии - частоты $f_{p k}^{(1)}$ и $f_{s k}$, взятые из таблицы.

Параметры нулевого отражения ТНz-волн от сенсоров с $d=240 \mu \mathrm{m}$

\begin{tabular}{|c|c|c|c|c|}
\hline Пленка & Поляризация & $k$ & $b_{p k}^{(1)}, b_{s k}, \operatorname{deg}$ & $f_{p k}^{(1)}, f_{s k}, \mathrm{THz}$ \\
\hline \multirow{5}{*}{$\mathrm{Si}_{1}$} & \multirow{5}{*}{$p$} & 1 & 64.32 & 0.223 \\
\hline & & 2 & 64.78 & 0.402 \\
\hline & & 3 & 64.60 & 0.586 \\
\hline & & 4 & 64.36 & 0.773 \\
\hline & & 5 & 64.18 & 0.960 \\
\hline \multirow{2}{*}{ PP } & \multirow[b]{2}{*}{$s$} & 0 & 63.09 & 0.223 \\
\hline & & 1 & 41.52 & 0.659 \\
\hline \multirow{5}{*}{$\mathrm{Si}_{2}$} & \multirow{5}{*}{$p$} & 1 & 66.06 & 0.223 \\
\hline & & 2 & 65.98 & 0.401 \\
\hline & & 3 & 65.48 & 0.586 \\
\hline & & 4 & 64.94 & 0.772 \\
\hline & & 5 & 64.46 & 0.959 \\
\hline
\end{tabular}

волны и быстрой моды с вещественной безразмерной постоянной распространения $\beta<1$, которая совпадает с $\beta_{p k}^{(1)}$, либо с $\beta_{s k}$, поскольку $r_{\alpha}(\beta)=0$ является дисперсионным уравнением для этой моды [16]. Высокая чувствительность сенсоров параметров сред, использующих резонансное возбуждение волноводных мод при условии их фазового синхронизма с падающей волной, хорошо известна в стандартной волноводной спектроскопии, где с помощью призм связи возбуждаются медленные моды пленочных волноводов [17]. Для рассматриваемых быстрых мод роль такой призмы выполняет воздух. Возбуждение этих мод имеет аналогию с возбуждением моды Ценнека, которое происходит при отражении волны $p$ поляризации, падающей под углом Брюстера на границу раздела прозрачных сред [10].

Сходство рассматриваемого сенсорного эффекта с аналогичными эффектами в волноводной спектроскопии, где используется зависимость от параметров окружающей среды провалов в угловой, либо частотной зависимостях отражательной способности призмы связи (так называемых темных т-линий), реализующихся при резонансном возбуждении волноводных мод [17], очевидно из рис. 5. Согласно рисунку, наличие примеси глюкозы в воде приводит к ощутимому изменению отражательных способностей сенсоров только в окрестности резонансных частот, соответствующих минимумам зависимостей 1 , а при заметном отклонении $f$ от $f_{p 1}^{(1)}$ на рис. $5, a$, либо от $f_{s 0}$ на рис. $5, b$ сенсорный эффект практически исчезает.

На рис. 4 и 5 наблюдается качественное различие характеристик отражательных сенсоров, использующих волны $p$ - и $s$-поляризации. При появлении в воде глюкозы в сенсоре на $p$-волнах происходит сдвиг минимума отражательной способности сенсора на шкале частот (рис. 5,a), тогда как в сенсоре на $s$-волнах основным эффектом является изменение величины отражательной способности сенсора (рис. 5, $b \mid /$ ). Кроме того, существенный сенсорный эффект при использовании $s$-волн наблюдается в гораздо более узком частотном интервале, чем в случае $p$-волн (рис. 4,5 ). 

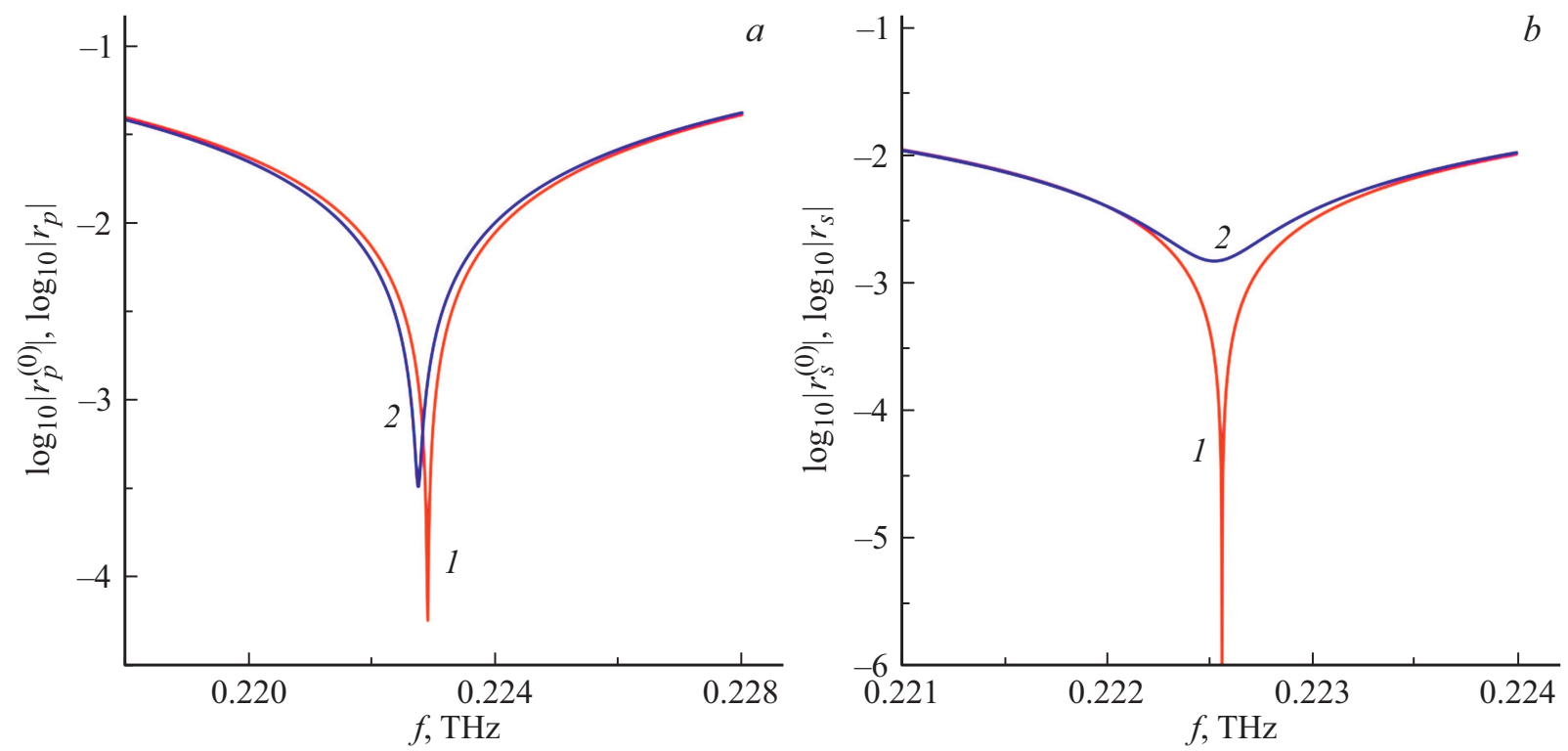

Рис. 5. Спектры отражательной способности сенсоров раствора глюкозы с пленкой $\mathrm{Si}_{1}$ толщины $d=240 \mu \mathrm{m}$ при $\theta=b_{p 1}^{(1)}=64.32^{\circ}$ для волн $p$-поляризации $(a)$ и с пленкой РР толщины $d=240 \mu \mathrm{m}$ при $\theta=b_{z 0}=63.09^{\circ}$ для волн $s$-поляризации (b). Кривые 1 - чистая вода, 2 - водный раствор глюкозы с $C=10 \mathrm{~g} / 1$.

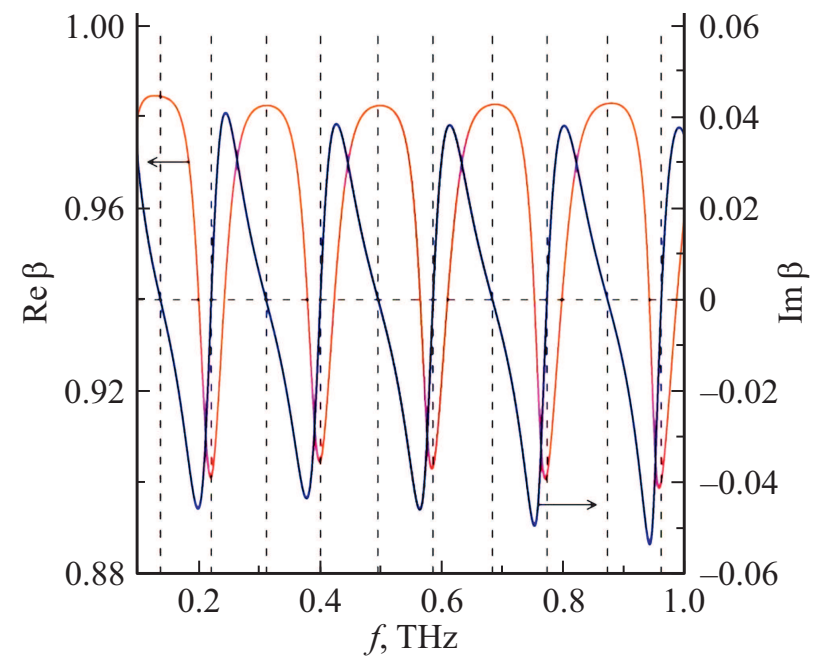

Рис. 6. Дисперсионные кривые моды р-поляризации структуры воздух-(пленка $\mathrm{Si}_{1}$ толщины $\left.d=240 \mu \mathrm{n}\right)$-вода. Вертикальные штриховые линии - частоты $f_{p k}^{(1)}$, горизонтальная штриховая линия - нулевой уровень $\operatorname{Im} \beta$.

Отметим также, что ощутимое отклонение параметра $\rho_{s}$ от 1 при выбранном угле падения $b_{s 0}$ наблюдается только в окрестности частоты $f_{s 0}$ (рис. $\left.4, b\right)$. В то же время аналогичные отклонения от 1 параметра $\rho_{p}$ имеют место в окрестности всех пяти табличных частот $f_{p k}^{(1)}$ (рис. 4, $a$ ) несмотря на то, что выбранный угол обращает систему $(6),(7)$ в тождество только в паре с $f_{p 1}^{(1)}$.

Последняя особенность объясняется дисперсионными свойствами быстрой моды $p$-поляризации на комплексной плоскости переменной $\beta$. Их иллюстрирует рис. 6, который построен в результате решения относительно $\beta$ дисперсионного уравнения для данной моды $r_{p}(\beta, f)=0$. Расчет выполнен методом контурного интегрирования [18].

Дисперсионные кривые на рис. 6 непрерывно переходят из физической $(\operatorname{Im} \beta<0)$ в нефизическую область $(\operatorname{Im} \beta>0)$. В момент перехода $(\operatorname{Im} \beta \rightarrow 0)$ на частотах $f=f_{p k}^{(l)}$ рассматриваемая мода допускает возбуждение при условии $\sin \theta=\operatorname{Re} \beta(f)=\beta_{p k}^{(l)}$. Набор решений 2 системы уравнений $(6),(7)$ формируется координатами точек пересечения кривой $\operatorname{Re} \beta(f)$ с вертикальными штриховыми линиями, расположенных в области $\operatorname{Re} \beta>0.96$, а набор решений 1 - координатами аналогичных точек, расположенных в области $\operatorname{Re} \beta<0.92$.

Как видно из рис. 6, названные точки практически совпадают с экстремальными точками зависимости $\operatorname{Re} \beta(f)$. Поэтому, дифференцируя дисперсионное уравнение $r_{p}(\beta, f)=0$ и используя приближение $\operatorname{Re}(d \beta)=0$, получаем

$$
\begin{aligned}
& \operatorname{Re}\left(\frac{\partial r_{p}}{\partial f}\right) d f-\operatorname{Im}\left(\frac{\partial r_{p}}{\partial \beta}\right) \operatorname{Im}(d \beta) \\
& +i\left[\operatorname{Re}\left(\frac{\partial r_{p}}{\partial \beta}\right) \operatorname{Im}(d \beta)+\operatorname{Im}\left(\frac{\partial r_{p}}{\partial f}\right) d f\right]=0
\end{aligned}
$$

где производные вычисляются при $\beta=\beta_{p k}^{(l)}, f=f_{p k}^{(l)}$. Комплексное уравнение (11) эквивалентно однородной системе двух вещественных алгебраических уравнений относительно $\operatorname{Im}(d \beta)$ и $d f$, определитель которой

$$
\operatorname{Re}\left(\frac{\partial r_{p}}{\partial f}\right) \operatorname{Re}\left(\frac{\partial r_{p}}{\partial \beta}\right)+\operatorname{Im}\left(\frac{\partial r_{p}}{\partial f}\right) \operatorname{Im}\left(\frac{\partial r_{p}}{\partial \beta}\right)=0
$$


С другой стороны, разложение в ряд Тейлора функции двух вещественных переменных $\left|r_{p}(\beta, f)\right|^{2}$ в окрестности точки с координатами $\beta=\beta_{p k}^{(l)}, f=f_{p k}^{(l)}$, по степеням приращений $\Delta \beta=\beta-\beta_{p k}^{(l)}, \Delta f=f-f_{p k}^{(l)}$, с точностью до квадратичных членов имеет вид

$$
\begin{aligned}
& \left|r_{p}(\beta, f)\right|^{2}=\left\{\left[\operatorname{Re}\left(\frac{\partial r_{p}}{\partial \beta}\right)\right]^{2}+\left[\operatorname{Im}\left(\frac{\partial r_{p}}{\partial \beta}\right)\right]^{2}\right\}(\Delta \beta)^{2} \\
& +\left\{\left[\operatorname{Re}\left(\frac{\partial r_{p}}{\partial f}\right)\right]^{2}+\left[\operatorname{Im}\left(\frac{\partial r_{p}}{\partial f}\right)\right]^{2}\right\}(\Delta f)^{2} \\
& +2\left[\operatorname{Re}\left(\frac{\partial r_{p}}{\partial f}\right) \operatorname{Re}\left(\frac{\partial r_{p}}{\partial \beta}\right)+\operatorname{Im}\left(\frac{\partial r_{p}}{\partial f}\right) \operatorname{Im}\left(\frac{\partial r_{p}}{\partial \beta}\right)\right] \Delta \beta \Delta f .
\end{aligned}
$$

Из (12) следует, что матрица квадратичной формы (13) относительно переменных $\Delta \beta$ и $\Delta f$ является диагональной. Следовательно, при малом $|\Delta \beta|$, или при малом модуле отклонении угла падения излучения на сенсор $\theta$ от $b_{p k}^{(l)}$, минимум отражательной способности сенсора, а значит и максимум его чувствительности, будут наблюдаться на частоте $f=f_{p k}^{(l)}$, что и объясняет вид зависимости $\rho_{p}(f)$ на рис. 4, $a$. Очевидно, что аналогичный результат будет иметь место и для зависимости $\rho_{p}(\theta)$ при фиксации частоты. Это обстоятельство может быть использовано для максимизации чувствительности сенсоров отражательного типа с перестраиваемым углом падения $\theta$, использующих монохроматический источник излучения. Заметим, что при рассмотрении волн $s$-поляризации матрица квадратичной формы, аналогичной (13), не диагональна.

\section{2. Эксперимент}

Исследован сенсор концентрации глюкозы в воде отражательного типа. В нем одна из стенок кюветы, в которую заливался исследуемый раствор, представляла собой полированную пластину из высокоомного кремния (пленка $\mathrm{Si}_{2}$ ). Площадь поверхности пластины составляла $50 \times 15 \mathrm{~mm}$ при диаметре THz-пучка $10 \mathrm{~mm}$. Использована типовая установка THz-TDS $[1,12]$. В ней фемтосекундные оптические импульсы Ti:Sa-лазера с частотой следования $80 \mathrm{MHz}$ преобразовывались в $\mathrm{THz}-$ импульсы кристаллом ZnTe толщиной $2 \mathrm{~mm}$. Пространственный диаметр THz-импульса $p$-поляризации на выходной грани кристалла был около $0.5 \mathrm{~mm}$. Импульс пропускался через линзу из кристаллического кварца с фокусным расстоянием $5 \mathrm{~cm}$, фокальная плоскость которой совмещалась с выходной гранью кристалла ZnTe. Нормаль к фронту коллимированного THz-излучения составляла угол $\theta$ с поверхностью пленки $\mathrm{Si}_{2}$. Изменение $\theta$ контролировалось с погрешностью $0.02^{\circ}$. Абсолютная погрешность задания $\theta$ порядка $0.5^{\circ}$ была вызвана дифракционной расходимостью THz-импульса. Рабочий угол $\theta$ настраивался по наиболее контрастной картине распределения $\left|r_{p}^{(0)}(f)\right|$. Излучение, отраженное от сенсора, проходило через еще одну линзу, идентичную названной выше. В ее задней фокальной плоскости помещалась широкоапертурная гиперсферическая кремниевая линза в виде полусферы радиуса $7.5 \mathrm{~mm}$, которая фокусировала излучение на фотопроводящую антенну (THz-приемник) с площадью рабочей поверхности $50 \times 50 \mu \mathrm{m}$. Моменты срабатывания приемника задавались с помощью управляемой линии задержки детектирующих лазерных импульсов и отстояли друг от друга на 0.05 ps. Регистрируемая напряженность электрического поля при каждом положении линии задержки усреднялась на интервале времени $0.1 \mathrm{~s}$. Поскольку метод THz-TDS предполагает деление фурье-образов импульсов, отраженных от сенсора, контактирующего с исследуемым раствором и с чистой водой, главное значение для получения достоверного отклика сенсора имеет стабильность угла $\theta$, а не его абсолютная величина. Поэтому с целью сохранения неизменной юстировки сенсора в ходе измерений перезаливка кюветы исследуемым раствором осуществлялась непосредственно в спектрометре. Измерения проводились на оптическом столе с виброизоляцией. Термостабилизация обеспечивалась на уровне $0.5^{\circ} \mathrm{C}$, при котором влияние термооптических эффектов было пренебрежимо малым по сравнению с сенсорным эффектом при $C \geq 10 \mathrm{~g} / 1$.

Перед тестированием сенсора были уточнены параметры пластинки $\mathrm{Si}_{2}$. Для этого по методике, описанной в [15], был измерен спектр модуля коэффициента пропускания пластины $\left|t_{f}(f)\right|$ с использованием $\mathrm{THz}-$ импульса, падающего нормально ее поверхности при нахождении пластины в воздухе. Для получения $t_{f}(f)$ фурье-образ прошедшего через пластину импульса делился на фурье-образ импульса, отраженного от металлического зеркала в отсутствие образца. Обработка распределения $\left|t_{f}(f)\right|$ методом наименьших квадратов с использованием дисперсионной модели Друде для диэлектрической проницаемости кремния [19] и известного выражения для $t_{f}$ [6] позволила установить толщину пластины $\mathrm{Si}_{2}$ и ее диэлектрическую проницаемость:

$$
\begin{gathered}
d=238.6 \mu \mathrm{m}, \\
\varepsilon_{f}=\varepsilon_{\infty}\left\{1-u^{2}\left[1+i(u F)^{-1}\right]\left(1+F^{2}\right)^{-1}\right\},
\end{gathered}
$$

где $\varepsilon_{\infty}=11.848, \quad u=7.57 \cdot 10^{-3}, \quad F=f f_{n}^{-1}, \quad f_{n}=$ $=0.992 \mathrm{THz}$.

О согласии экспериментальных и расчетных данных для функции $\left|t_{f}(f)\right|$, полученных в рамках модели (14), и о дисперсионных характеристиках пластинки $\mathrm{Si}_{2}$ позволяет судить рис. 7.

Экспериментальные данные для реакции сенсора на изменение в водном растворе глюкозы ее концентрации $C$ представлены на рис. 8 . 

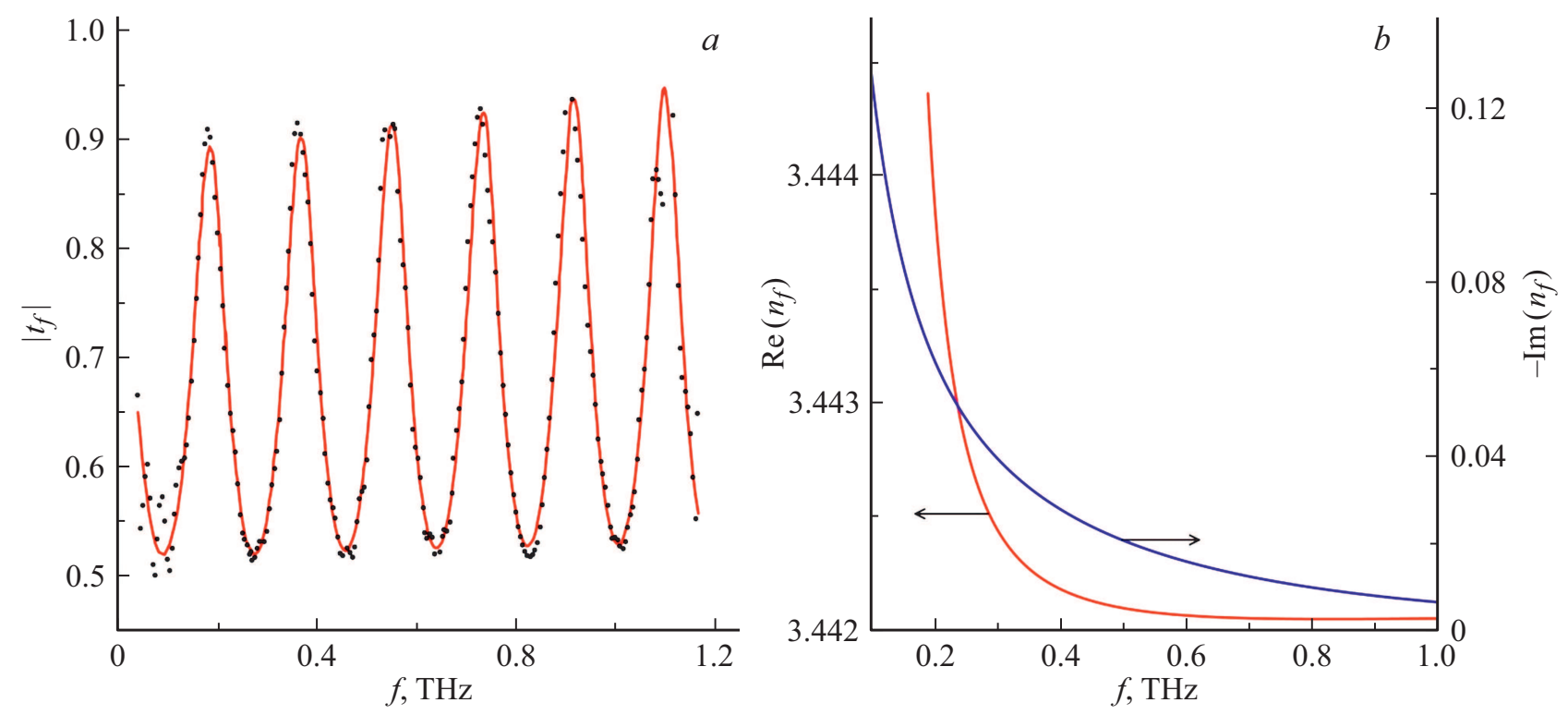

Рис. 7. Характеристики кремниевой пластины $\mathrm{Si}_{2}$, использованной в эксперименте: $a$ - частотная зависимость модуля коэффициента пропускания пластины в воздушном окружении (дискретные точки - эксперимент, сплошная кривая - теория); $b$ - спектр комплексного показателя преломления пластины $n_{f}=\sqrt{\varepsilon_{f}}$ (модель (14)).
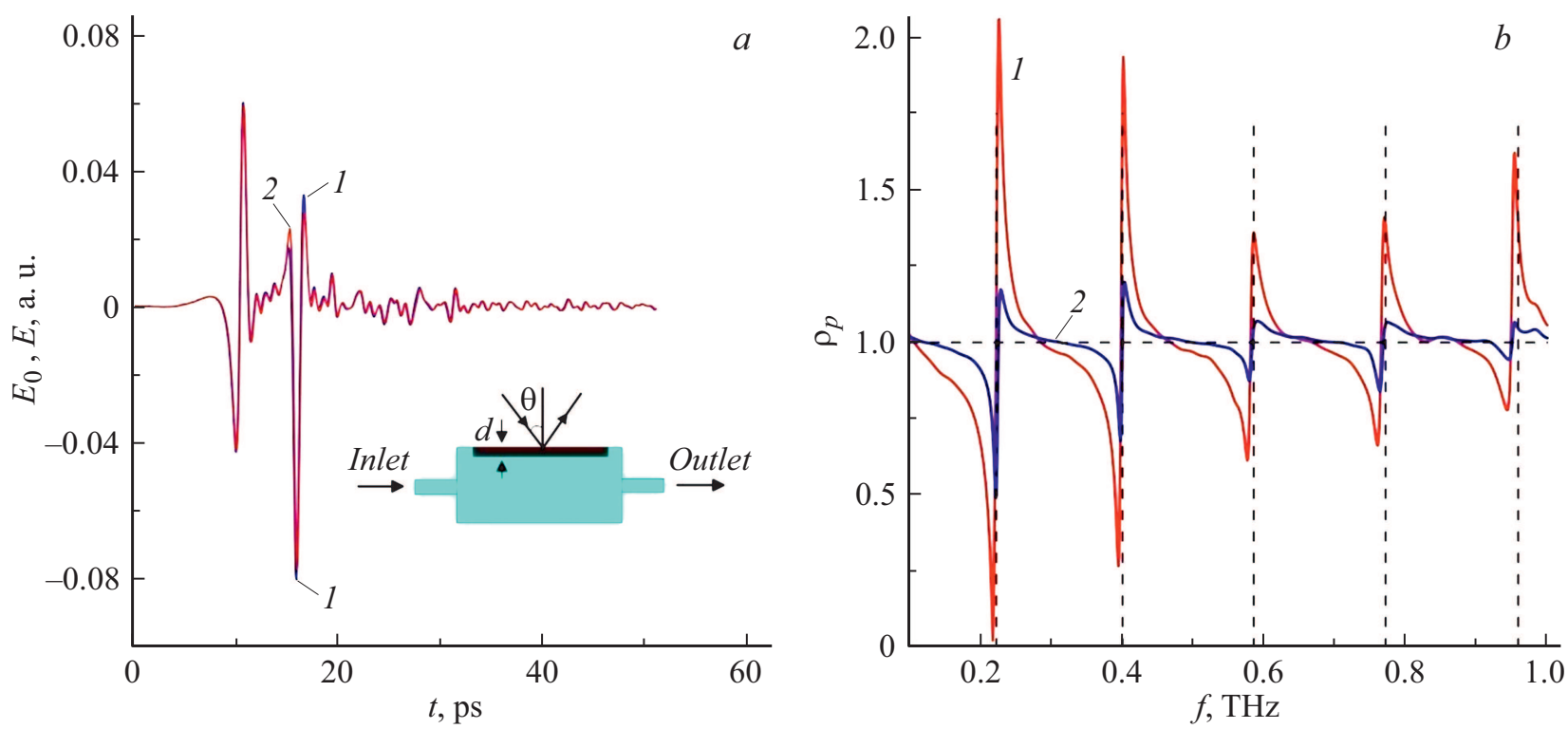

Рис. 8. Экспериментальный отклик сенсора концентрации глюкозы в водном растворе: $a$ - ТНz-импульсы, отраженные от сенсора с пластинкой $\mathrm{Si}_{2}$ (схема его чувствительного элемента - на вставке) при заполнении кюветы чистой водой (кривая 1 ) и раствором глюкозы с $C=30 \mathrm{~g} / 1(2) ; b-$ спектры параметра (15): горизонтальная штриховая линия $-C=0 \mathrm{~g} / 1$, сплошная кривая $1-C=30 \mathrm{~g} / 1,2-C=10 \mathrm{~g} / \mathrm{l}$; вертикальные штриховые линии - расчетные частоты для пленки $\mathrm{Si}_{2}$, взятые из таблицы.

Здесь зависимости $\rho_{p}(f)$ оценены по формуле

$\rho_{p}=\left|\int_{t_{1}}^{t_{2}} E(t) \exp (-i 2 \pi f t) d t\right|\left|\int_{t_{1}}^{t_{2}} E_{0}(t) \exp (-i 2 \pi f t) d t\right|^{-1}$,

где $t_{1}=0 \mathrm{ps}$ и $t_{2}=52 \mathrm{ps}-$ границы временного промежутка, использованного при измерении импульсов, $E(t)$ - напряженность электрического поля в отра- женном от сенсора импульсе при значении концентрации $C ; E_{0}(t)=\left.E(t)\right|_{C=0 \text { g/l. }}$ Интегралы в (15) рассчитаны методом трапеций. Расчеты и измерения выполнены при $\theta=65.90^{\circ}$.

Согласно рис. $8, a$, влияние небольшой примеси глюкозы в растворе слабо влияет на временную форму импульса, но существенно сказывается на его фурье-образе. Заметим, что часть импульса при $t \leq 12$ рs определяется отражением излучения от границы раздела пластина- 
воздух и практически не зависит от $C$. Наиболее информативным является интервал времени $15-25 \mathrm{ps}$, в котором перекрываются импульсы, отраженные от границ раздела пластина-воздух и пластина-раствор. Осцилляции сигналов при $t>25 \mathrm{ps}$ можно связать с резонансным поглощением THz-излучения водяным паром, присутствовавшим в атмосфере лаборатории.

Графики зависимостей $\rho_{p}(f)$ на рис. $8, b$ качественно подобны графику $\rho_{p}(f)$ на рис. $4, a$. В частности, изломы всех графиков наблюдаются на частотах $f_{p k}^{(1)}$. Однако экспериментальные распределения $\rho_{p}(f)$ в окрестности частот $f_{p k}^{(1)}$ (рис. $\left.8, b\right)$ более уширены по сравнению с их теоретическим аналогом (рис. $4, a)$ (в частности, при $C=10 \mathrm{~g} / 1$ модуль отклонения $\rho_{p}(f)$ от 1 в окрестности резонансной частоты $f_{p 1}^{(1)}=0.223 \mathrm{THz}$ превышает $5 \%$ в диапазонах $0.22 \leq f \leq 0.226 \mathrm{THz}$ на рис. 4, $a$ и $0.185 \leq f \leq 0.248 \mathrm{THz}$ на рис. $8, b)$. Это можно связать с дифракционной расходимостью THz-излучения, которая в теоретической модели не учитывалась, и с конечными пределами интегрирования в (15).

Отметим, что частоты $f_{p k}^{(1)}$ для пленок $\mathrm{Si}_{1}$ и $\mathrm{Si}_{2}$ практически совпадают, тогда как соответствующие им углы нулевого отражения $b_{p k}^{(1)}$ существенно различаются, причем их расхождение увеличивается с уменьшением номера $k$ (см. таблицу). Причина этих особенностей состоит в том, что в случае высокопреломляющих пленок существенное влияние на значения $b_{p k}^{(1)}$ оказывает коэффициент поглощения пленки [11]. Для пленки $\mathrm{Si}_{2}$ этот коэффициент $\left(\left|\operatorname{Im}\left(n_{f}\right)\right|\right)$ заметно превосходит, особенно в низкочастотной области, значение 0.005 для пленки $\mathrm{Si}_{1}$ (рис. $7, b)$, тогда как значения $d$ и $\operatorname{Re}\left(n_{f}\right)$, влияющие на $f_{p k}^{(1)}$, для обеих пленок, близки. Как показано в [11], подобные особенности могут быть использованы для измерения коэффициентов поглощения пленок.

\section{Заключение}

Как следует из рис. $8, b$, примеси глюкозы концентрации $C=10$ и $30 \mathrm{~g} / 1$ в воде отчетливо обнаружимы и различимы, что с применением других $\mathrm{THz}-$ сенсоров водных растворов труднодостижимо (например, в недавно опубликованной работе [20], где использован метод пропускания THz-изучения через раствор в кювете, заметное изменение отклика сенсора наблюдалось при шаге по концентрации глюкозы в растворе $100 \mathrm{~g} / \mathrm{l})$. Таким образом, предложенные сенсоры, использующие эффект нулевого отражения монохроматических волн от диссипативной структуры пленка-раствор, могут с

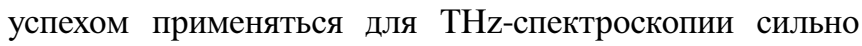
поглощающих водных растворов. Помимо высокой чувствительности к концентрации детектируемого компонента их достоинствами являются простота в изготовлении и высокая стабильность точек спектральных особенностей, положение которых определяется и мо- жет регулироваться толщиной диэлектрической пленки (пластины).

\section{Благодарности}

Авторы благодарны коллегам из МГУ им. М.В. Ломоносова за содействие в проведении экспериментов.

\section{Финансирование работы}

Работа выполнена в рамках задания 1.3.03 „Разработка теории методов оптического контроля наноразмерных тонкопленочных структур“ ГПНИ „Фотоника, оптои микро-электроника“, № госрегистрации 20161316 и гранта РФФИ 17-00-00270.

\section{Конфликт интересов}

Авторы заявляют, что у них нет конфликта интересов.

\section{Список литературы}

[1] А.А. Ангелуц, А.В. Балакин, М.Г. Евдокимов, М.Н. Есаулков, М.М. Назаров, И.А. Ожередов, Д.А. Сапожников, П.М. Солянкин, О.П. Черкасова, А.П. Шкуринов. Квант. электрон., 44 (7), 614 (2014). [A.A. Angeluts, A.V. Balakin, M.G. Evdokimov, M.N. Esaulkov, M.M. Nazarov, I.A. Ozheredov, D.A. Sapozhnikov, P.M. Solyankin, O.P. Cherkasova, A.P. Shkurinov. Quant. Electron., 44 (7), 614 (2014). DOI: 10.1070/qe2014v044n07abeh015565]

[2] O.A. Smolyanskaya, N.V. Chernomyrdin, A.A. Konovko, K.I. Zaytsev, I.A. Ozheredov, O.P. Cherkasova, M.M. Nazarov, J.-P. Guillet, S.A. Kozlov, Yu.V. Kistenev, J.-L. Coutaz, P. Mounaix, V.L. Vaks, J.-H. Son, H. Cheon, V.P. Wallace, Yu. Feldman, I. Popov, A.N. Yaroslavsky, A.P. Shkurinov, V.V. Tuchin. Prog. Quant. Electron., 62, 1 (2018). DOI: 10.1016/j.pquantelec.2018.10.001

[3] O. Cherkasova, M. Nazarov, A. Shkurinov. Opt. Quant. Electron., 48, 217 (2016). DOI: 10.1007/s11082-016-0490-5

[4] T. Torii, H. Chiba, T. Tanabe, Y. Oyama. Digital Health, 3, 1 (2017). DOI: $10.1177 / 2055207617729534$

[5] F. Lan, F. Luo, P. Mazumder, Z. Yang, L. Meng, Z. Bao, J. Zhou, Y. Zhang, S. Liang, Z. Shi, A.R. Khan, Z. Zhang, L. Wang, J. Yin, H. Zeng. Biomed. Opt. Express, 10 (8), 3789 (2019). DOI: 10.1364/BOE.10.003789

[6] М. Борн, Э. Вольф. Основы оптики (Наука, М., 1973)

[7] H.G. Tompkins, E.A. Irene. Handbook of Ellipsometry (William Andrew Publishing, Norwich, 2005)

[8] R.M.A. Azzam. J. Opt. Soc. Am. A, 30 (10), 1975 (2013). DOI: $10.1364 /$ JOSAA.30.001975

[9] P. Adamson. Appl. Opt., 53 (21), 4804 (2014). DOI: $10.1364 /$ ao. 53.004804

[10] А.В. Кукушкин, А.А. Рухадзе, К.З. Рухадзе. УФН, 182 (11), 1205 (2012). DOI: 10.3367/UFNr.0182.201211f.1205

[11] А.Б. Сотский, С.С. Михеев, М.М. Назаров. Докл. НАН Беларуси, 63 (6), 672 (2019). DOI: $10.29235 / 1561-8323-2019-63-6-672-679$

[12] J. Neu, C.A. Schmuttenmaer. J. Appl. Phys., 124, 231101 (2018). DOI: $10.1063 / 1.5047659$ 
[13] M.M. Nazarov, O.P. Cherkasova, A.P. Shkurinov. Quant. Electron., 46 (6), 488 (2016). DOI: 10.1070/QEL16107

[14] J.-L. Coutaz, F. Garet, E. Bonnet, A.V. Tishchenko, O. Parriaux, M. Nazarov. Acta Phys. Pol. A, $107(1), 26$ (2005). DOI: 10.12693/aphyspola.107.26

[15] M.M. Nazarov, A.V. Shilov, K.A. Bzheumikhov, Z.Ch. Margushev, V.I. Sokolov, A.B. Sotsky, A.P. Shkurinov. IEEE Trans. Terahertz Sci. Technol., 8(2), 183 (2018). DOI: 10.1109/TTHZ.2017.2786030

[16] А.Б. Сотский, L.M. Steingart, J.H. Jackson, П.Я. Чудаковский, Л.И. Сотская. ЖТФ, 83 (11), 105 (2013).

[17] А.В. Хомченко. Волноводная спектроскопия тонких пленок (БГУ, Минск, 2002)

[18] А.Б. Сотский. Теория оптических волноводных элементов (МГУ им. А.А. Кулешова, Могилев, 2011)

[19] E.D. Palik. Handbook of Optical Constants of Solids (Academic Press, Orlando, Florida, 1985)

[20] Г.Р. Мусина, А.А. Гавдуш, Н.В. Черномырдин, И.Н. Долганова, В.Э. Улитко, О.П. Черкасова, В.Н. Курлов, Г.А. Командин, И.В. Животовский, В.В. Тучин, К.И. Зайцев. Опт. и спектр., 128 (7), 1020 (2020). DOI: 10.21883/OS.2020.07.49576.65-20 